\title{
Parabola Error Evaluation Based on Geometry Optimizing Approxima- tion Algorithm
}

\author{
Lei Xianqing ${ }^{1, *}$, Gao Zuobin ${ }^{1}$, Wang Haiyang ${ }^{2}$ and Cui Jingwei ${ }^{3}$ \\ ${ }^{1}$ Henan University of Science and Technology, Luoyang, 471003, China \\ ${ }^{2}$ Heavy Industries Co., Ltd, Luoyang, 471003, China \\ ${ }^{3}$ Luoyang Bearing Science \&Technology Co. Ltd, Luoyang, 471003, China
}

\begin{abstract}
A more accurate evaluation for parabola errors based on Geometry Optimizing Approximation Algorithm (GOAA) is presented in this paper. Firstly, according to the least squares method, two parabola characteristic points are determined as reference points to form a series of auxiliary points according to a certain geometry shape, and then, assumption ideal auxiliary parabolas are reversed by the parabola geometric characteristic. The range distance of all given points to the assumption ideal parabolas are calculated by the auxiliary parabolas as supposed ideal parabolas, and the reference points, the auxiliary points, reference parabola and auxiliary parabolas are reconstructed by comparing the range distances, the evaluation for parabola error is finally obtained after repeating this entire process. On the basis of $0.1 \mathrm{~mm}$ of a group of simulative metrical data, compared with the least square method, while the criteria of stop searching is 0.00001 $\mathrm{mm}$, the parabola error value from this algorithm can be reduced by $77 \mu \mathrm{m}$. The result shows this algorithm realizes the evaluation of parabola with minimum zone, accurately and stably.
\end{abstract}

Keywords: Error evaluation, geometry approximation, minimum zone, parabola error.

\section{INTRODUCTION}

In the Image Processing or the Computer Graphics, the fitting of conic plays a foundational and important role in the curve profile matching [1]. Now that, parabola is difficult to fit as its geometry has conic feature. In recent years, parabola is applied in many fields of Mechanical design and parts measurement algorithm. For example, among the aviation and aerospace industry, the parabola type of radial flow impeller is adopted widely in the radial flow impeller machine of propulsion system in order to enhance the aerodynamic performance and strength of the impeller [2]. At present, a mature and representative algorithm to evaluate parabola profile error is not used in most of coordinate measuring machine $(\mathrm{CMM})$. Therefore, to evaluate parabola profile error accurately has important application value for design and processing of aerospace parts and precision measurement methods.

So far, there is not a unified standard for parabola profile error at home and broad. GB/T 1184-1996 does not have a specialized criterion to judge parabola profile error. Parabola fitting methods can be divided into two types: the methods based on algebraic distance and the methods based on geometric distance. In 2001, the parabola fitting of least-squares orthogonal distance (LOD) method was presented by Sung Joon Ahn, and the method measured better than the least squares techniques, but the iterations of the calculations is relatively complex. Ahn's method [3] improved the efficiency of iteration by processing the weighted fitting coefficient matrix. Zhang [4] and Strum [5] proposed the objective function based on the bias of geometry distance. But, the computation process contains a $4^{\text {th }}$ order equation. It is not stable to construct an ideal conic in some conditions. Liu [1] used six different constraints to get six basic conics, and generate the best conic by adding certain weights to the coefficients of the six basic conics. Different fitting methods with constrain matrices were listed for specific types of conic in Harker's paper [6]. Chernov [7] posted a weighted gradient fitting method to the scattered data points, and pointed out that the method can relieve the complexity in some conditions. Gao [8] combined the Genetic Algorithm and least square method together to solve the conic fitting problems. Li [9] mainly talks about how to select the coordinate system and the constraints to make the fitting curve approximately closer with the implicit conic equation. But her method is needed to transfer the coordinate system first.

All in all, the works just mentioned discussed the objective functions and the different constraints after transferring the coordinates. In this paper, we discuss two important problems. The one is to fit parabola in optional position of rectangular coordinate system, another is to evaluate the profile error of given data.

\section{THE PRINCIPLE OF THE GOAA}

Firstly, we can obtain two feature points (focus and intersection between axis and directrix) and initial profile error $d_{0}$ 


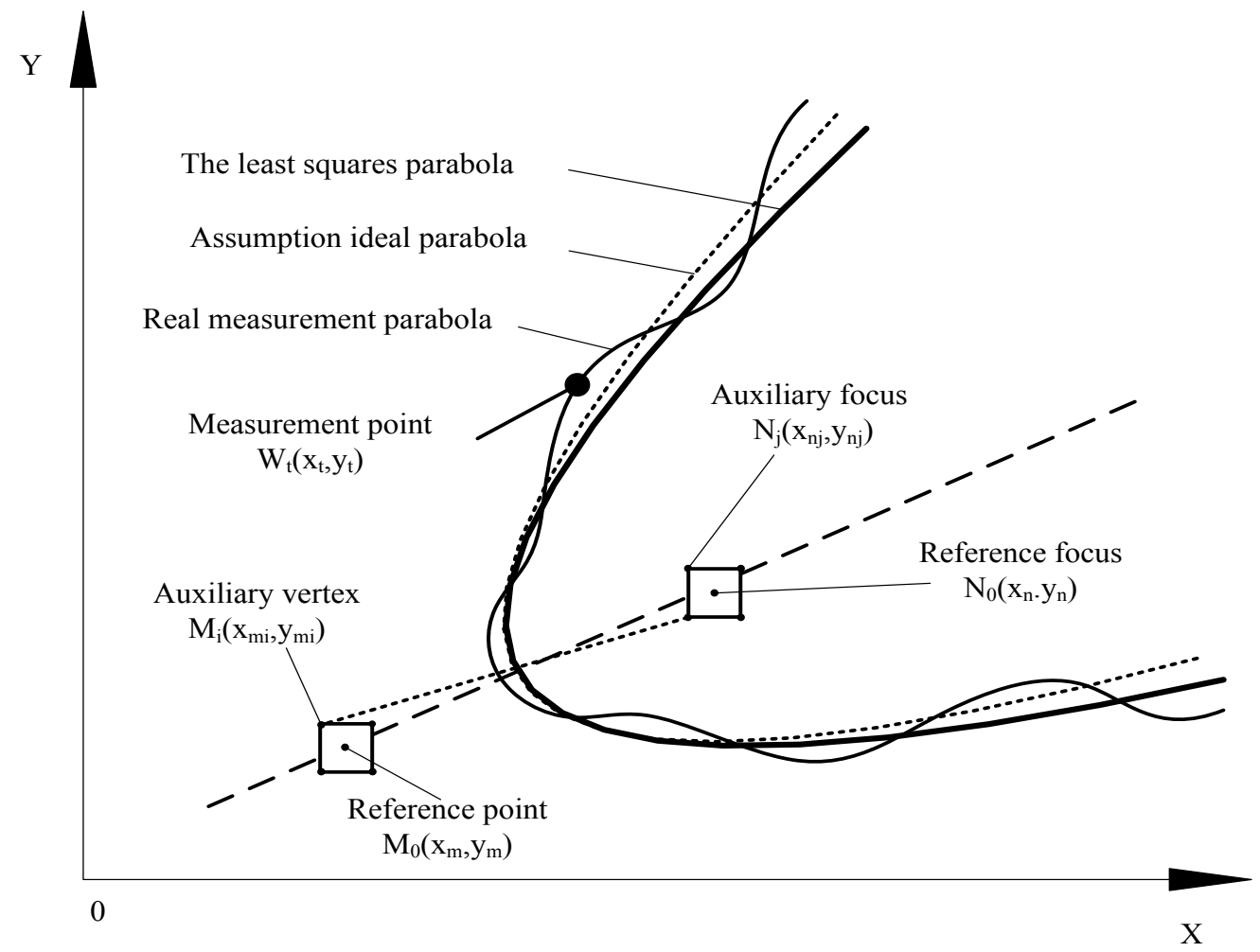

Fig. (1). The evaluation principle.

by least squares method (LSM) with constraints [10]. Then, the two feature points are treated as initial reference points, and then, 4 auxiliary points (vertexes of searching square) are arranged around them respectively (see Fig. 1), so we can get 16 groups of auxiliary feature points. If we assume arbitrary groups of auxiliary feature points are ideal feature points of ideal parabola 16, assuming ideal parabolas are reserved with geometry knowledge. Afterwards, calculating the shortest distances between given points and assuming ideal parabolas, the minimum distance is named $d_{1}$. If $d_{0} \leq d_{1}$, then the reference feature points remain and the length of searching square is reduced by half. Meanwhile, the new auxiliary feature points are reconstructed and repeat the process of solving $d_{1}$. If $d_{0}>d_{1}$, the feature points of assuming ideal parabola corresponding to $d_{1}$ are the new reference feature points and the length of searching square is remains the same, 16 groups of new auxiliary feature points are achieved. Repeating above processing, when the length of searching square reduces a certain value, we could consider that the assuming ideal parabola approach ideal parabola very closely and stop the searching process. So the minimum between $d_{0}$ and $d_{1}$ is the profile error of parabola.

\section{THE STEPS OF THE GOAA}

\subsection{Selecting the Initial Reference Points, Initial Length and Accuracy Parameter}

Assuming the measurement points are expressed by $W_{t}\left(x_{t}, y_{t}\right)(t=1,2,3, \ldots, N)$. In order to reserve 6 parameters of parabola expressed by conic equation, we set LSM feature points (focus $M_{0}\left(x_{m}, y_{m}\right)$ and intersection between axis and directrix point $\left.N_{0}\left(x_{n}, y_{n}\right)\right)$ of parabola as initial reference points according to plane analytic geometry knowledge. The length of searching square is LSM error or estimated parabolic error $d_{0}$. The precision parameter can be changed according to the effective number of measurement data, precision of instrument and accuracy requirement of measuring object, when the precision parameter is under micron level, $\delta=0.0001 \mathrm{~mm}$.

\subsection{Constructing Auxiliary Feature Points}

Two searching squares are constructed whose lengths $l$ have initial error $d_{0}$. At same time, their centers are initial reference points (that are feature points) $M_{0}\left(x_{m}, y_{m}\right)$ and $N_{0}\left(x_{n}, y_{n}\right)$ (see Fig. 1). The vertexes of searching squares are named auxiliary points $M_{i}\left(x_{m i}, y_{m i}\right)$ and $N_{j}\left(x_{n j}, y_{n j}\right)$ $(i=j=1,2,3,4)$, they are calculated below:

$\left\{\begin{array}{l}x_{m 1}=x_{m 2}=x_{m}+l / 2 \\ x_{m 3}=x_{m 4}=x_{m}-l / 2 \\ y_{m 1}=y_{m 4}=y_{m}+l / 2 \\ y_{m 2}=y_{m 3}=y_{m}-l / 2\end{array}\left\{\begin{array}{l}x_{n 1}=x_{n 2}=x_{n}+l / 2 \\ x_{n 3}=x_{n 4}=x_{n}-l / 2 \\ y_{n 1}=y_{n 4}=y_{n}+l / 2 \\ y_{n 2}=y_{n 3}=y_{n}-l / 2\end{array}\right.\right.$

\subsection{Constructing Auxiliary Parabolas}

The 16 groups of auxiliary feature points are obtained from auxiliary points $M_{i}\left(x_{m i}, y_{m i}\right)(i=1,2,3,4) \quad$ and 
$N_{j}\left(x_{n j}, y_{n j}\right)(j=1,2,3,4)$ according to permutation and combination knowledge. Next, the 16 groups of main parameters of parabolas are achieved by the equation (2).

$\left\{\begin{array}{l}p_{i j}=\left\|M_{i}-N_{j}\right\| \\ \left|x c_{i j}\right|=\left|\begin{array}{l}\left(x_{m i}+x_{n j}\right) / 2 \\ y c_{i j}\end{array}\right| \mid \begin{array}{l}\left(y_{m i}+y_{n j}\right) / 2 \\ \tan \left(\theta_{i j}\right)=\left(y_{m i}-y_{n j}\right) /\left(x_{m i}-x_{n j}\right)\end{array}\end{array}\right.$

Where, $p_{i j}$ are focal length, $\left(x c_{i j}, y c_{i j}\right)$ are vertexes, $\theta_{i j}$ are the rotation angles of assuming ideal parabolas.

The measured parabola in any position of plane can be expressed by $A x^{2}+B x y+C y^{2}+D x+E y+F=0$. The coefficients of the equation are gained with equation (3).

$$
\left\{\begin{array}{l}
A_{i j}=\cos ^{2} \theta_{i j} \\
B_{i j}=2 \sin \theta_{i j} \cos \theta_{i j} \\
C_{i j}=\sin ^{2} \theta_{i j} \\
D_{i j}=2\left(p_{i j} \sin \theta_{i j}-x c_{i j} \cos ^{2} \theta_{i j}-y c_{i j} \sin \theta_{i j} \cos \theta_{i j}\right) \\
E_{i j}=-2\left(x c_{i j} \sin \theta_{i j} \cos \theta_{i j}+y c_{i j} \sin ^{2} \theta_{i j}+p_{i j} \cos \theta_{i j}\right) \\
F_{i j}=\left(x c_{i j} \cos \theta_{i j}+y c_{i j} \sin \theta_{i j}\right)^{2} \\
\quad+2 p_{i j}\left(y c_{i j} \cos \theta_{i j}-x c_{i j} \sin \theta_{i j}\right)
\end{array}\right.
$$

In the equation (3), the 16 assuming ideal auxiliary parabola equations are reserved.

\subsection{Calculating the Range Distance from Given Points to Assuming Ideal Parabola}

Assuming that points $O_{s}\left(x_{s}, y_{s}\right)(s=1,2,3, \ldots, N)$ are orthogonal points between given points $W_{t}\left(x_{t}, y_{t}\right)$ $(t=1,2,3, \ldots, N)$ and assuming ideal auxiliary parabolas, so points $O_{s}\left(x_{s}, y_{s}\right)$ can be obtained by the reference [11].

$$
\left\{\begin{array}{l}
A_{i j} x_{s}^{2}+B_{i j} x_{s} y_{s}+C_{i j} y_{s}^{2}+D_{i j} x_{s}+E_{i j} y_{s}+F_{i j}=0 \\
\left(y_{s}-y_{t}\right)\left(2 A_{i j} x_{s}+B_{i j} y_{s}+D_{i j}\right)-\left(x_{s}-x_{t}\right)\left(B_{i j} x_{s}+\right. \\
\left.\left.2 C_{i j} y_{s}+E_{i j}\right)=0\right)
\end{array}\right.
$$

We can gain the range distances from given points to assuming ideal parabolas.

$d d_{i j s}= \pm\left\|O_{s}-W_{t}\right\|$

Where, the $d_{i j s}$ are positive value when given points locate out of assumption ideal parabola, otherwise, $d_{i j s}$ are negative value. The range distance from given points to assumption ideal parabola are obtained as follow:

$d_{i j}=\max \left\{d d_{i j s}\right\}-\min \left\{d d_{i j s}\right\}$ (7).

The minimum range distance is named $d_{1}$ with equation

$d_{1}=\min \left(d_{i j}\right)$

\subsection{Approximation Searching Method}

If $d_{0} \leq d_{1}$, searching squares are reconstructed with constant reference feature points and half of length, repeat steps 3.3-2.5, If $d_{0} \leq d_{1}$, the feature points of assuming ideal parabola corresponding to $d_{1}$ are the new reference feature points and the length of searching square is the error right now $l=d_{1}$, repeating steps 3.3-3.5, when the length of searching square reduces a certain value (general condition $l \leq 0.0001 \mathrm{~mm}$ ), we could consider that the assuming ideal parabola approaches ideal parabola very closely and stop the searching process. So the minimum value between $d_{0}$ and $d_{1}$ is the profile error of parabola $\Delta d$.

$\Delta d=\min \left(d_{0}, d_{1}\right)$

\section{EXAMPLE}

There is a series of measurement points belonging to the optional position parabola in the plane, added some noise points whose pre-set error is $0.1 \mathrm{~mm}$ and are then simulated to test the algorithm in Table 1. The initial reference points (come from the feature points of the LSM [11]) are $M_{0}(-$ $0.5287,1.5344) \mathrm{mm}$ and $N_{0}(1.5419,-5.5361) \mathrm{mm}$, meanwhile, the initial length of searching square are $l=0.3 \mathrm{~mm}, l=0.3$ $\mathrm{mm}$ and $l=0.09 \mathrm{~mm}$ respectively, stop searching condition are $l \leq 0.001 \mathrm{~mm}, l \leq 0.0001 \mathrm{~mm}$ and $l \leq 0.00001 \mathrm{~mm}$ separately, Using the GOAA, the results are in Table 2 and the process of the successive approximations are shown in Fig. (2-4).

We can see from the Table 2 , the result of the GOAA are very close to the pre-set error with the same stop searching condition in spite of different initial zone.

It can be seen from Fig. (2-4) that, under the different initial length, the GOAA can snap out of the local extreme and rapidly converge to the global minimum. In addition to the same initial length, when stop searching conditions are $l \leq 0.001 \mathrm{~mm}, l \leq 0.0001 \mathrm{~mm}$ and $l \leq 0.00001 \mathrm{~mm}$, the errors are smaller and smaller, that is, the smaller the values of the stop searching condition is, the more precise the evaluation is, which shows that the evaluating accuracy of the GOAA depends on the pre-set stop searching condition and the GOAA has good stability, monotonic convergence. Generally, the stop searching condition $l \leq 0.00001 \mathrm{~mm}$ is totally satisfied for the assessment requirements in micron level measurement.

\section{CONCLUSION}

This paper studies the parabola profile error of minimum zone evaluation based on Geometry Optimizing Approximation 
Table 1. Measurement data (mm).

\begin{tabular}{|c|c|c|}
\hline No & $\mathbf{x}$ & $\mathbf{y}$ \\
\hline 1 & -10 & 5 \\
\hline 2 & -9.96464 & 5.035355 \\
\hline 3 & -9 & 4.05 \\
\hline 4 & -8.53238 & 3.574403 \\
\hline 5 & -7 & 2.45 \\
\hline 6 & -5.5241 & 1.468689 \\
\hline 7 & -3 & 0.45 \\
\hline 8 & -2.51213 & 0.263993 \\
\hline 9 & -2 & 0.2 \\
\hline 10 & -1.5 & 0.1125 \\
\hline 11 & -0.99502 & 0.099752 \\
\hline 12 & 0 & 0 \\
\hline 13 & 1 & 0.05 \\
\hline 14 & 1.990194 & 0.249029 \\
\hline 15 & 2.5 & 0.3125 \\
\hline 16 & 3.516518 & 0.565307 \\
\hline 17 & 4.5 & 1.0125 \\
\hline 18 & 4.977639 & 1.294721 \\
\hline 19 & 5.5 & 1.5125 \\
\hline 20 & 6.527249 & 2.070578 \\
\hline 21 & 7 & 2.45 \\
\hline 22 & 9.534437 & 4.47625 \\
\hline 23 & -20 & 20 \\
\hline 24 & 20 & 20 \\
\hline
\end{tabular}

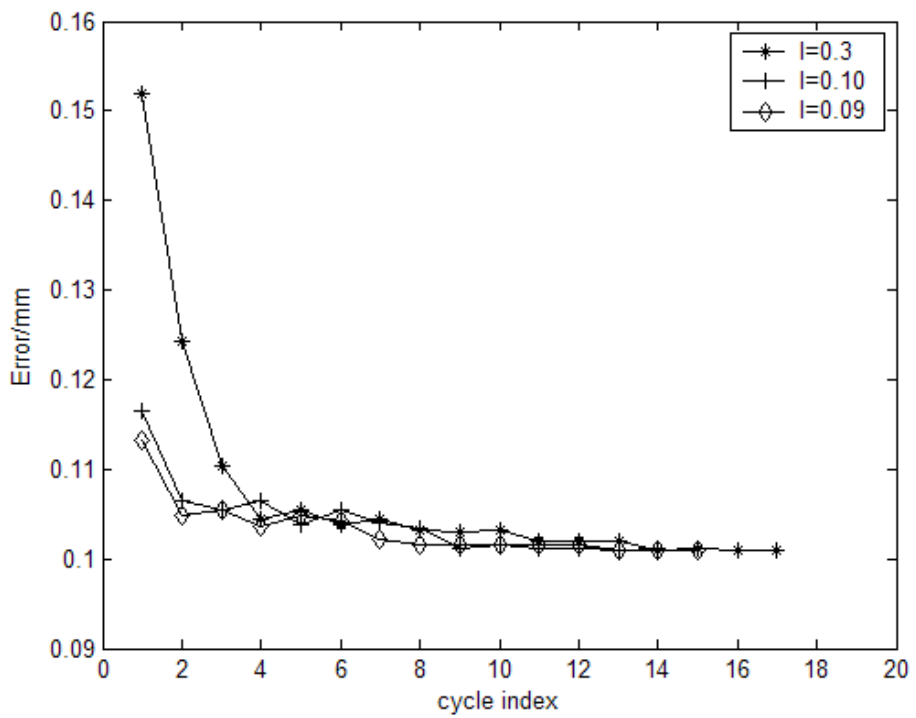

Fig. (2). The process of successive approximations under the $l \leq 0.001 \mathrm{~mm}$. 


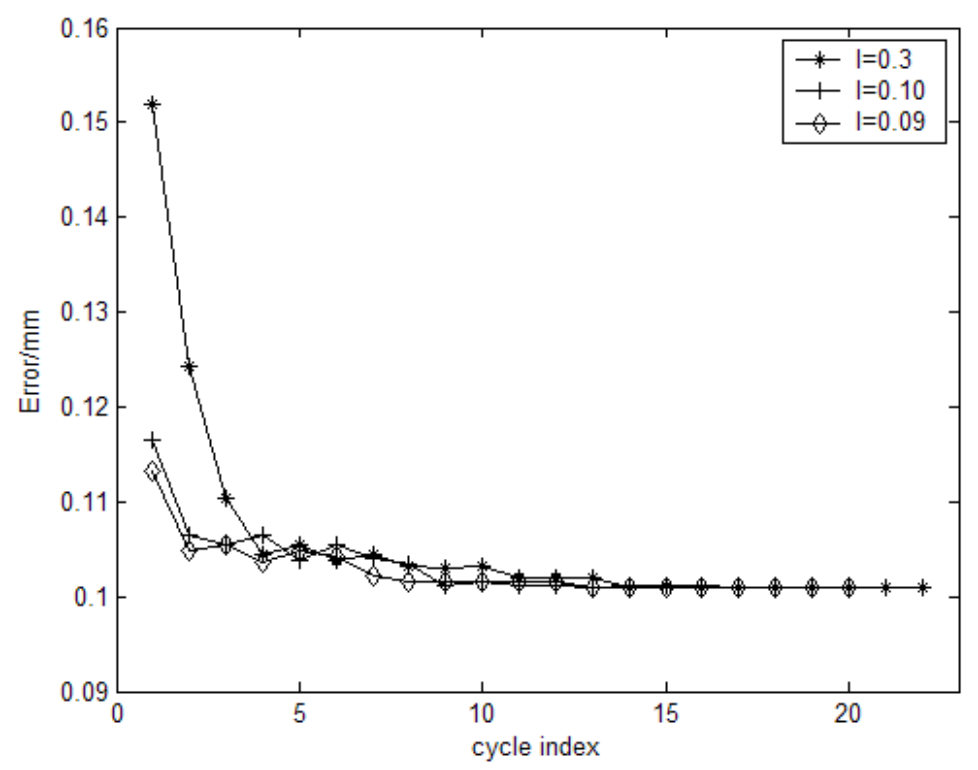

Fig. (3). The process of successive approximations under the $l \leq 0.0001 \mathrm{~mm}$.

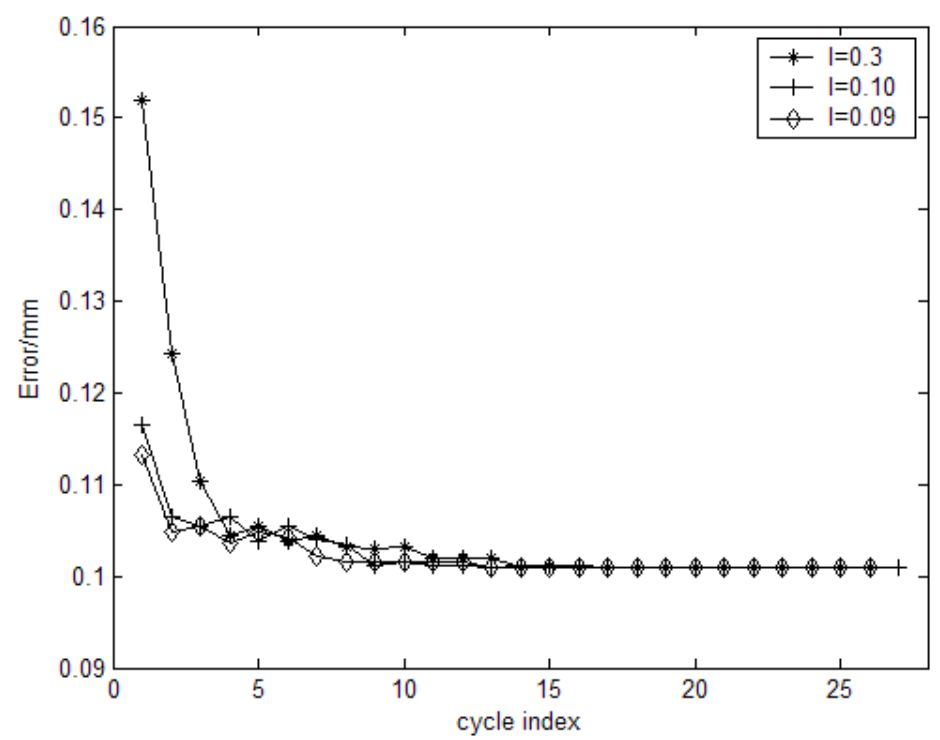

Fig. (4). The process of successive approximations under the $l \leq 0.00001 \mathrm{~mm}$

Table 2. The results $(\mathrm{mm})$.

\begin{tabular}{|c|c|c|c|}
\hline \multirow{2}{*}{ Initial } & \multicolumn{3}{|c|}{ Stop Searching Condition } \\
\cline { 2 - 4 } & $\boldsymbol{l} \leq \mathbf{0 . 0 0 1}$ & $\boldsymbol{l} \leq \mathbf{0 . 0 0 0 1}$ & $\boldsymbol{l} \leq \mathbf{0 . 0 0 0 0 1}$ \\
\hline \hline$l=0.3$ & 0.1009557 & 0.1008201 & 0.1008134 \\
\hline$l=0.10$ & 0.1009054 & 0.1008912 & 0.1008882 \\
\hline$l=0.09$ & 0.1009838 & 0.1009271 & 0.1008914 \\
\hline
\end{tabular}

Algorithm according to plane analytic geometry knowledge. This algorithm is not depended on uniformity of measurement points and fulfills process simply. A group of simulated measurement points whose error is $0.1 \mathrm{~mm}$ are evaluated with GOAA. The error reduces to $72 \mu \mathrm{m}$ than the LSM [11] when the stop searching condition is $l \leq 0.00001 \mathrm{~mm}$. The results show that GOAA can evaluate profile error of parabola fast and accurately. Meanwhile, this algorithm provides a certain degree of reference value for evaluation of parabola profile error. 


\section{CONFLICT OF INTEREST}

The authors confirm that this article content has no conflict of interest.

\section{ACKNOWLEDGEMENTS}

The authors gratefully acknowledge Basic and Frontier Technology research proposal of Henan Province (No.122300410114, 1223004132), the Ph. D. Initial Foundation of Henan University of Science \&Technology, Scientific Research Innovation Ability Cultivation Foundation of Henan University of Science\& Technology (No.2010CZ0002) and Innovation Scientists and Technicians Troop Construction Projects of Henan Province for financial support of this research work.

\section{REFERENCES}

[1] R. Zimbelman, "A Contribution to the Problem of CementAggregate Bond", Cement and Concrete Research, vol. 15, pp. 801-8, June 1985.

[2] H. X. Liu, C. M. Zhang, and X. X. Liang, "Fitting Conic to Scattered Data in a Plane", Journal of Computer Aided Design \& Computer Graphics, vol. 16, pp. 1594-1598, Nov 2004, (in Chinese).
[3] H. Späth, "Least-squares orthogonal distances fitting of parabolas", in Proceedings of the IMACS-GAMM International Symposium on Numerical Methods and Error-Bonds, 1996, pp. 261-269.

[4] S. J. Ahn, W. Rauh, and H. Warnecke, "Least-squares orthogonal distances fitting of circle, sphere, ellipse, hyperbola, and parabola", Pattern Recognition, vol. 34, pp .2283-2303. Dec 2001.

[5] Z. Y. Zhang, "Parameter estimation techniques: A tutorial with application to conic fitting", Image and Vision Computing Journal, vol. 15, pp. 6-18. Jan 1997.

[6] P. Sturm, and P. Gargallo, "Conic fitting using the geometric distance", in Proceedings Computer Vision - ACCV $2007-8^{\text {th }}$ Asian Conference on Computer Vision vol. 4844LNCS, pp. 784-795, 2007.

[7] M. Harker, and P. Leary, "Direct type-specific conic fitting and eigenvalue bias correction", Image and Vision Computing, vol.26, pp. 372-381, March 3, 2008

[8] N. Chernov, and C. Lesort, "Statistical efficiency of curve fitting algorithms", Computational Statistics and Data Analysis, vol. 47, pp. 713-728, Nov 2004.

[9] S. Gao, and C. Li, "An improved genetic algorithm for solving conic fitting problems", in Proceedings World Congress on Computer Science and Information Engineering, pp. 800-804, 2009.

[10] P. Li, "A New Method of Fitting Conic to Scattered Date", in Proceedings-2010 $3^{\text {rd }}$ International Congress on Image and Signal Processing, vol. 6, pp. 2722-2726, 2010.

[11] H. Y. Wang, "Evaluation Algorithm of the Form Error of Parabolic Profile Based on Least Squares Method", Manufacturing Automation, vol. 35, p. 23-25, Jun 2013. (in Chinese).

(C) Xianqing et al.; Licensee Bentham Open.

This is an open access article licensed under the terms of the Creative Commons Attribution Non-Commercial License (http://creativecommons.org/licenses/by-nc/3.0/) which permits unrestricted, non-commercial use, distribution and reproduction in any medium, provided the work is properly cited. 